\title{
Perte Post-Récolte Dans La Perspective De Stockage Des Bulbes D'oignons (Allium Cepa L.) En Milieu Paysan Dans Le Département De La Bénoué Nord- Cameroun
}

\author{
Housseini Djida Jacques, \\ Alioum Paul Sounou, \\ Saïfoullah Daïrou,
}

Institut de Recherche Agricole pour le

Développement (IRAD), Station Polyvalente de Recherche Agricole de Garoua SPRA-Garoua, Université de Ngaoundéré, Faculté des Sciences, Département de Sciences Biologiques

Doi:10.19044/esj.2020.v16n18p124 URL:http://dx.doi.org/10.19044/esj.2020.v16n18p124

\section{Résumé}

Le caractère saisonnier de la production de l'oignon de variété Violet de Galmi en irrigation dans le département de la Bénoué limite essentiellement sa disponibilité sur les marchés durant toute l'année. L'objectif de cette étude est d'évaluer les pertes post-récoltes dans une perspective de stockage des bulbes d'oignons de variété Violet de Galmi en milieu paysan dans la localité de Garoua, Nord-Cameroun. Sur un échantillon de 55 bulbes d'oignons choisi au hasard dans le magasin d'un paysan, les paramètres suivants ont été enregistrés toutes les deux semaines et pendant quatre mois de conservation : le poids des bulbes et le dénombrement de bulbes pourris et germés. Pendant toute la période de conservation le poids de bulbes décroit graduellement. Les bulbes pourris représentaient la perte la plus importante 9,1 pourcents, suivi de perte de masse soit 6,9 pourcents et les bulbes germés 5,5 pourcents. Le taux de perte globale pendant toute la période de stockage est de 21,5 pourcents.

Mots clés : Perte post-récolte, stockage, Violet de Galmi, Nord-Cameroun 


\title{
Post-Harvest Loss in the Perspective of Onions Bulb (Allium Cepa L.) Storage in a Farming Environment in Benoue Department of North-Cameroon
}

\author{
Housseini Djida Jacques, \\ Alioum Paul Sounou, \\ Saïfoullah Daïrou,
}

Institute of Agricultural Research for Development (IRAD), Multipurpose Station of Agricultural Research of Garoua, University de Ngaoundere, Faculty of Sciences, Departement of Biological Sciences

\begin{abstract}
The seasonal nature of the production of the Violet Galmi onion in irrigation in the Bénoué department essentially limits its availability on the markets throughout the year. The objective of this study is to assess postharvest losses with a view to storing bulbs of the Violet de Galmi variety onions in a farming environment in the locality of Garoua, North-Cameroon. On a sample of 55 onion bulbs chosen at random from a farmer's store, the following parameters were recorded every two weeks and for four months of storage: the weight of the bulbs and the count of rotten and sprouted bulbs. During the entire storage period, the weight of the bulbs gradually decreases. Rotten bulbs represented the largest loss of 9.1 percent, followed by mass loss of 6.9 percent and sprouted bulbs 5.5 percent. The overall loss rate during the entire storage period is 21.5 percent.
\end{abstract}

Keywords: Post-Harvest Loss, Storage, Violet Galmi, North-Cameroon

\section{Introduction}

L'oignon (Allium cepa L) appartient à la famille des Liliaceae, à laquelle appartiennent également le poireau, l'ail et l'échalote. La caractéristique de cette famille est la présence d'un bulbe formé par un renflement plus ou moins important au niveau de la base des feuilles qui sert d'organe de réserve (FAO 1996). Le Nigeria occupe la $6{ }^{\text {ème }}$ position mondiale de pays producteur d'oignon avec une production de plus de 618000 tonnes en 2007 (Sulumbe et al., 2015). En Afrique centrale et au Cameroun en particulier, sa production est d'environ 90000 tonnes avec un rendement moyen de 10 t/ha, dont $85 \%$ de la production nationale proviennent des 
régions du Nord et de l'Extrême-nord (Kamga et al., 2016). C'est une culture de rente importante après le coton et l'arachide et la première spéculation maraîchère du pays (Yaya et al., 2003). Les bulbes d'Allium cepa constituent un légume apprécié et un élément essentiel de nombreuses sauces africaines (Schmelzer et Gurid-Fakim, 2013). Les bulbes sont mangés comme légumes cuits, crus ou frits. On estime qu'en moyenne au Cameroun, la consommation individuelle est d'environ 1 kilogramme par mois (Essang et al., 2002). La production d'oignon de la variété violet de Galmi est essentiellement saisonnière. Cela influence tout son circuit de commercialisation. A côté de cela s'ajoute les techniques de productions, de récoltes et de conservation inappropriées. Bien que l'oignon soit considéré comme cultures semi périssables, les pertes après récolte peuvent être importantes surtout dans les régions de forte production. Les pertes après récolte peuvent atteindre $40 \%$ de la production et le long transport vers les marchés du Sud-Cameroun contribue à augmenter ces pertes (Cathala et al., 2003). Des données exactes sur la nature et l'étendue des pertes dans les grands bassins de production au Cameroun ne sont pas actualisés. La production massive d'oignon dans les régions du Nord et Extrême-Nord du Cameroun incite les producteurs et commerçants à conserver les excès de production au lieu de les vendre à des prix dérisoires. Ainsi, ils assureront un approvisionnement régulier des marchés et pourront faire des profits. Pour entreprendre un tel projet une évaluation des pertes pendant toute la période de stockage est nécessaire. C'est dans cette perspective que la présente étude a pour but d'évaluer les pertes post-récoltes des bulbes d'oignons de variété Violet de Galmi durant quatre mois de stockage en milieu paysan.

\section{Matériels et méthodes \\ Zone d'étude}

La Bénoué est un département de la région du nord Cameroun et couvre environ $13,614 \mathrm{Km}^{2}$. Son climat est du type soudano-sahélien qui s'étend au-delà du $10^{\circ}$ de latitude nord. Elle se caractérise par une longue saison sèche de sept à neuf mois et des précipitations peu abondantes variant de 900 à $300 \mathrm{~mm} / \mathrm{an}$ du sud vers le nord. La température moyenne dépasse $28^{\circ}$ C (FAO, 2005).

Tableau 1 : Température et humidité relative durant expérimentation.

\begin{tabular}{llllll}
\hline \hline Mois & $\begin{array}{l}\text { Température } \\
\max \text { en }{ }^{\circ} \mathbf{C}\end{array}$ & $\begin{array}{l}\text { Température } \\
\min \text { en }{ }^{\circ} \mathbf{C}\end{array}$ & $\begin{array}{l}\text { Température } \\
\text { moyenne en }{ }^{\circ} \mathbf{C}\end{array}$ & $\begin{array}{l}\text { Humidité } \\
\%\end{array}$ & en \\
\hline Mai & 38 & 29 & 34 & 60 \\
Juin & 36 & 27 & 31 & 73 \\
Juillet & 35 & 25 & 30 & 80 \\
Août & 32 & 24 & 28 & 87 & \\
\hline \hline
\end{tabular}

(Source : historique-météo.net 2020) 


\section{Conduite culturale}

La variété Violet de Galmi se caractérise par ses qualités organoleptiques appréciables, homogénéité du calibrage, sa coloration violette, son habillage des tuniques, sa fermeté, et son aptitude à la conservation (Habou et al., 2015). La culture a été mise en place en zone périurbaine sous irrigation le long des berges de la Bénoué Nord-Cameroun. Le semis a été effectué le 11/11/2018, le repiquage en champs le 22/12/2018 et la récolté le 01/04/2019. Un apport en fertilisant minéral de $600 \mathrm{~kg}$ de 2010-10 de N.P.K. à l'hectare a été effectué en deux temps en vue de rehausser le niveau de fertilité de ce sol fortement dégradé.

\section{Dispositif et condition de conservation}

Après la récolte des bulbes, un premier tri a été effectué afin de retirer tous les bulbes blessés ou présentant des débuts de pourritures. Puis les bulbes saints et de calibres homogènes sont introduits dans le magasin de stockage d'un paysan de la localité. Les bulbes étaient disposés sur des nattes de paille en couche inférieur à trois centimètres. Deux semaines plus tard un tri est à nouveau effectué afin d'éliminer les bulbes ayant subis des chocs ou stress pendant le transport et manutention. Pour notre expérimentation, un échantillon de 55 bulbes a été choisi au hasard, puis marqués et les données suivantes ont été enregistrées toutes les deux semaines pendant quatre mois : le poids ( $\mathrm{g}$ ) de chaque bulbe et le dénombrement de bulbes pourris et germés. Les taux de perte ont également été évalués selon la formule suivante :

Taux de perte $=($ Nombre de bulbe pourris ou germé $/$ Nombre total de bulbe $)$ X 100

\section{Résultats et discussions}

\section{Evolution du poids moyen des bulbes}

La figure 1 illustre les pertes de poids pendant les quatre mois de conservation des bulbes. Les deux premiers mois de conservation de Mai $\left(34^{\circ} \mathrm{C}\right)$ à début Juillet $\left(30^{\circ} \mathrm{C}\right)$, le poids moyen des bulbes chute brutalement de $111,1 \mathrm{~g}$ à $104,8 \mathrm{~g}$. Ensuite de Juillet $\left(30^{\circ} \mathrm{C}\right)$ à Août $\left(28^{\circ} \mathrm{C}\right)$ le poids moyen des bulbes décroit plutôt graduellement de 104,8 g à 103,4 g. Ces pertes de poids moyen des bulbes enregistrées durant toute la période de l'étude pourraient être dues aux variations de températures durant toute la période de notre expérimentation. Dans l'étude de Jolayemi et al., (2018) des pertes quantitatives d'humidités des bulbes sont également observées en milieu contrôlé et ces pertes croissent lorsque les températures croissent. Sharma et al., (2014) recommandent de maintenir dans l'environnement de stockage une température en-dessous de $30^{\circ} \mathrm{C}$ pour limiter les pertes de poids. 


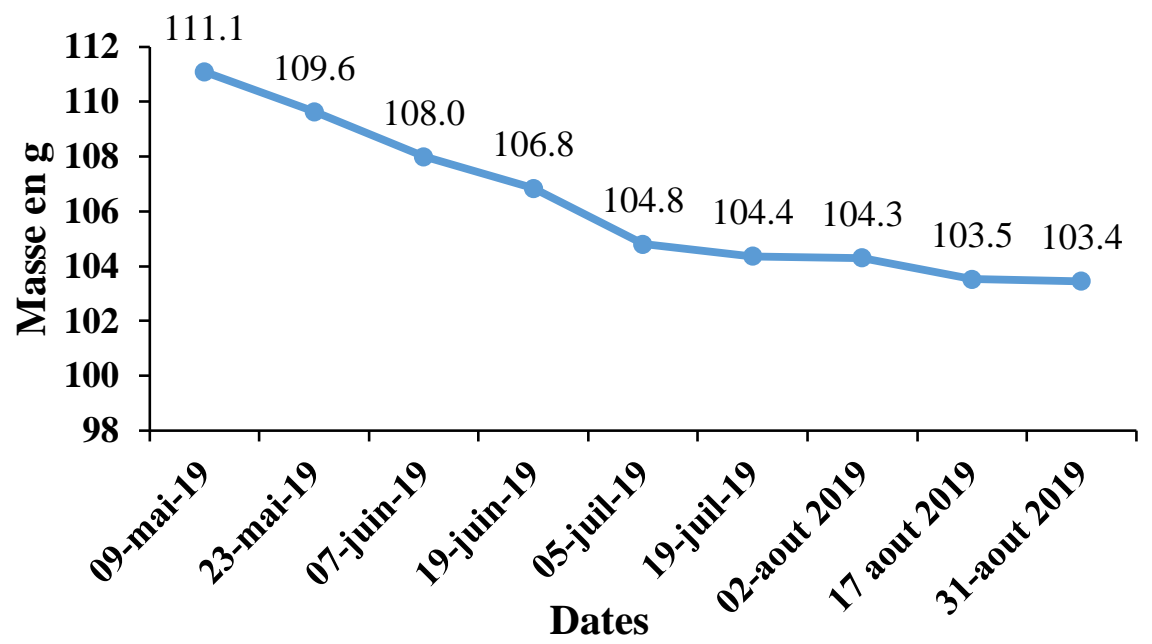

Figure 1 : Evolution des poids moyens des bulbes durant toute la période de stockage.

\section{Types de perte et comportement des bulbes en stockage}

Il ressort de la figure 2 que la courbe de pourriture des bulbes présente deux pics donc le plus important est observé en Mai immédiatement en début de stockage et le moins important autour du 5 Juin. L'apparition des symptômes de pourriture en début d'entreposage a été également observée par Fondio et al., (2001). Cela pourrait être une preuve que les agents d'origine fongiques et bactériologiques responsables de ces pourritures proviendraient du champ. Les premiers bulbes germés apparaissent en fin Juin et présentent un pic en début Juillet. Lorsque les bulbes sont récoltés, ils sont dormants et la germination a lieu lorsque les conditions deviennent favorables (Sarrad, 2017). Pour les pertes de masses des bulbes, elles s'étalent sur toute la période de stockage et sont les plus importantes de Mai à début Juillet puis décroisent graduellement dans le temps. Ces pertes dépendent certes des conditions de stockage, mais pourraient également être liés aux conduites culturales. Les mêmes types de pertes ont été relevés par Habou et al., (2015) dans un contexte d'apports d'azotes sur l'aptitude de conservation de bulbe d'oignon. 


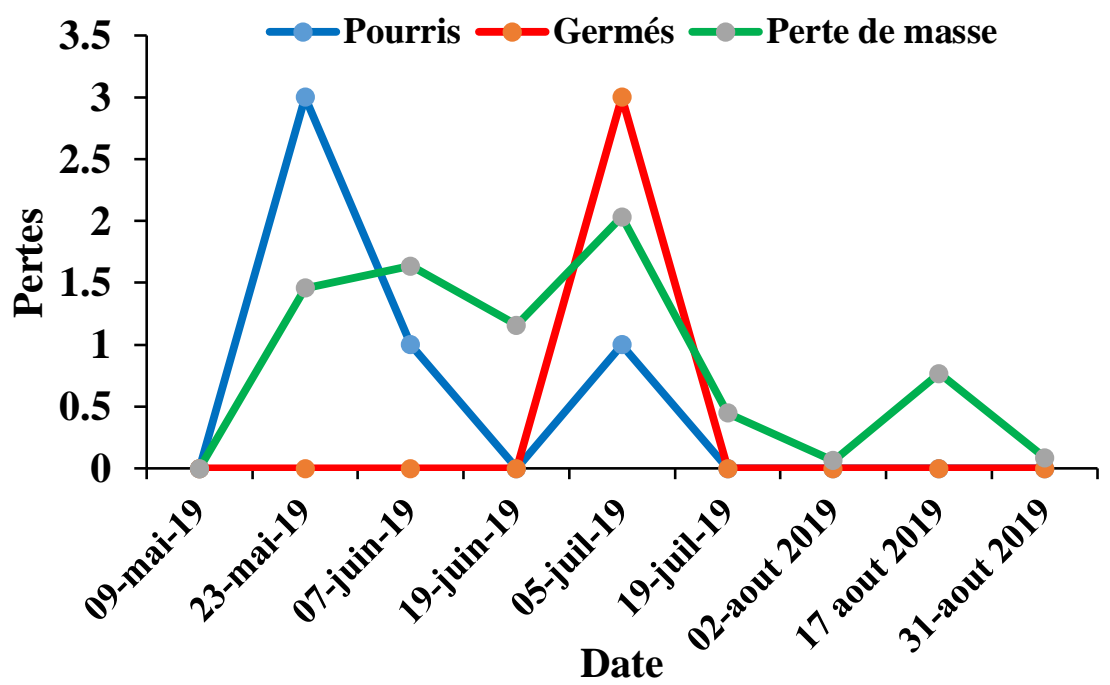

Figure 2 : Evolution des différents types de pertes en stockage.

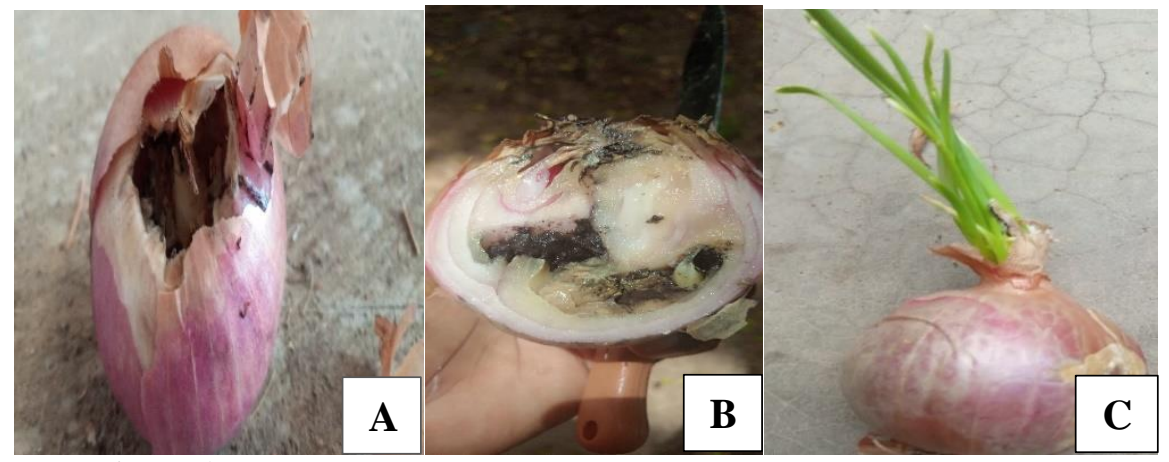

Figure 3 : Pourriture des bulbes en A \& B, germination de bulbe en C.

\section{Taux de pertes}

Les bulbes pourris (Figure 3 A \& B) sont les plus importants et représentent $9,1 \%$ des pertes, suivis des pertes de masse avec un taux de 6,9 $\%$ et enfin les bulbes germés (Figure $3 \mathrm{C}$ ) avec un taux de 5,5\% (Figure 4). Les pertes globales durant les quatre mois de stockage sont de 21,5\% (Figure 4). Des taux de pertes plus importants 16 à $33 \%$ ont été enregistrés dans l'étude de Habou et al., (2015) dues à la pourriture et à la germination des bulbes. Selon les travaux de Claudine et al., (2013), des apports d'engrais azotés augmentent le rendement, réduisent le taux de matière sèche, accroissent la pourriture et la germination précoce, limitant leurs aptitudes à la conservation après la récolte. Ko et al., (2012) soulignent également qu'il existe une corrélation positive entre l'aptitude générale à la conservation de l'oignon et la teneur en matière sèche des bulbes. 


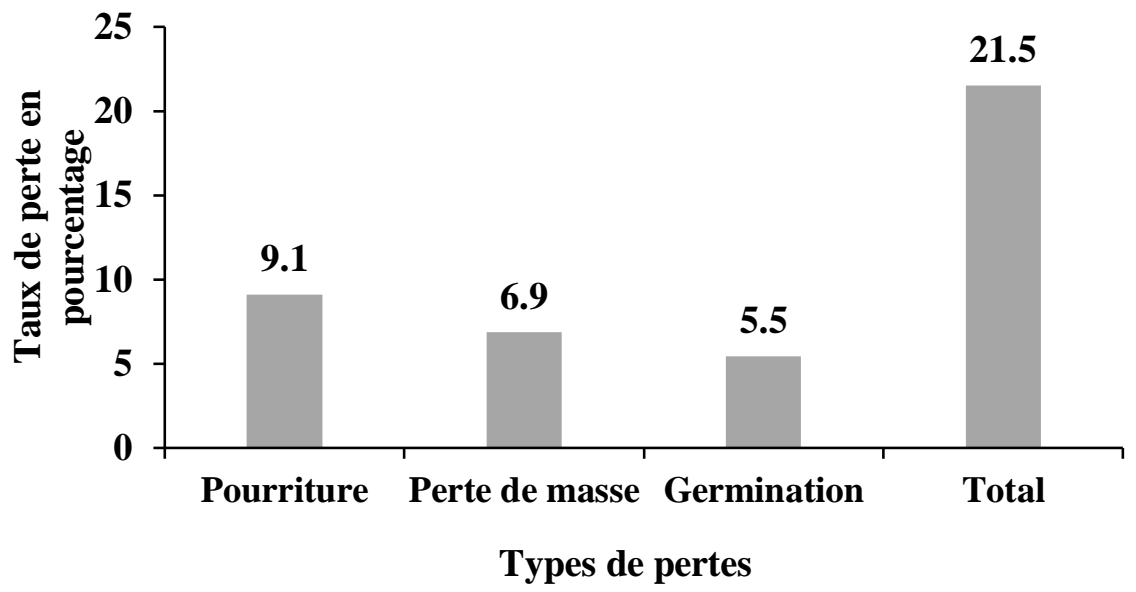

Figure 4 : Taux de perte post-récolte des bulbes d'oignons.

\section{Conclusion}

La filière oignon représente un atout économique majeur dans les régions du Nord et Extrême-Nord du Cameroun, mais de nombreuses contraintes le long de la filière restent à être levées. Une conservation inappropriée peut engendrer des pertes considérables pour les producteurs. Pour la variété Violet de Galmi, durant quatre mois de stockage, la pourriture des bulbes représente la perte les plus importantes, suivie de la perte de masse qui s'étend sur toute la période de stockage et enfin les bulbes germés. Le taux de perte globale enregistré est de 21,5 pourcents. Des facteurs tels qu'une fertilisation riche en azote, le choix variétal, la température de l'environnement de stockage, la densité d'entreposage des bulbes, la fréquence des tris... peuvent agir fortement sur l'aptitude de conservation des bulbes et nécessitent d'avantage d'investigation.

\section{References :}

1. Cathala, M., Woin, N., Essang, T. (2003). L'oignon, une production en plein essor en Afrique sahélo-soudanienne: cas du NordCameroun. Cahiers Agricultures. 12 : 261-6.

2. Claudine, K., Paulo, R. E., Volnei, P., Francisco, O. G. M. J., Joao, V. N. (2013). Yield and conservation of onions affected by nitrogen fertilization in no-tillage system. Hortic. Bras., 31(4): 559-567.

3. Essang, T. H., Woin, N., Andoune, B. E. (2002). Stratégies et comportements des acteurs économiques de la filière oignon. Actes du colloque Prasac de Garoua «Savanes africaines: des espaces en mutation, des acteurs face à de nouveaux défis ». 13p.

4. FAO (1996). Techniques de production de semences d'oignon au Sénégal. Coopération Régionale pour le Développement des 
Productions Maraîchères en Afrique de l'Ouest - Composante Sénégal GCP/SEN/033/BEL.

5. FAO (2005). L'irrigation en Afrique en chiffre - Enquête AQUASTAT. Cameroun.

6. Fondio, L., Kouame, C., Djidji, A. H. (2001). Evaluation du comportement de quelques variétés d'oignon (Aluim cepa L.) au champ et en stockage à Ferkessedougou. Agronomie Africaine. 13(3) : 113-130.

7. Habou, Z. A., Mahamadou, C. I., Adam, T. (2015). Effet de l'azote sur l'aptitude à la conservation des bulbes d'oignon (Allium cepa L.). International Journal of Biological and Chemical Sciences., 9(6): 2889-2896, 2015

8. http://www.historique-météo.net 2020.

9. Jolayemi, O. S., Nassarawa, S. S., Lawal, O. M., Sodipo, M. A., Oluwalana, I. B. (2018). Monitoring the changes in chemical properties of red and white onions (Allium cepa) during storage. Journal of Stored Products and Postharvest Research. 9(7): 7886.

10. Kamga, R. T., Tchouamo, I. R., Chendjou, R., Bidogeza J. C., Afari-Sefa V. (2016). Gender inequality in smallholder onion (allium cepa 1.) production in the far north region of Cameroon, Journal of Gender, Agriculture and Food Security, 1, (3): 85-103.

11. Ko, S., Chang, W. N., Wang, J. F., Cherng, S. J., Shanmugasundaram, S. (2012). Storage variability anong short-day onion cultivars under high temperature and high relative humidity, and its relationship with disease incidence and bulb charateristic.J. Amer. Soc. Hort. Sci. 127(5): 848-854.

12. Sarrad, M., (2017). http ://www.agri-mag.com/conservationtraditionnelle-de-loignon-dans-la-province-de-hajed. 2020.

13. Schmelzer et Gurib-Fakim, (2013). Plantes Médicales 2 (Vol 11) Ressources végétales de l'Afrique Tropicale.

14. Sharma, K. Ko, E. Y., Assefa, A. D., Ha, S., Nile, S. H., Lee, E. T., Park, S. W. (2014). Temperature-dependent studies on the total phenolics, flavonoids, antioxidant activities, and sugar content in six onion varieties. Journal of Food and Drug Analysis. 23(2):243-252.

15. Sulumbe, I. M., Shettima, B. G., John, T. B. (2015). An analysis of the marketing of onion in Monguno local government area of Borno State, Nigeria. Journal of Marketing and Consumer Research 13:9- 14.

16. Yaya, M., Noé, W., Magalie, C. (2003). Les producteurs d'oignons du Nord-Cameroun: les défis d'une filière à la quête d'une place au soleil, archives-ouvertes. 8p. ffhal-00143301ff. 